\title{
The 2009 framework for undergraduate medical education in the Netherlands
}

\author{
R.F.J.M. Laan, R.R.M. Leunissen, C.L.A. van Herwaarden (on behalf of the Project Group)*
}

\begin{abstract}
Summary
The 2009 Framework defines the joint Dutch national learning outcomes to be attained by medical students after completing their three-year master programme in medicine. The Framework thus helps to guarantee to society at large and to patients in particular that medical graduates who are starting out as practitioners have attained a certain professional level. This level is the aggregate of the physicians' target profile, physicians' (sub-) competencies to be achieved and the list of issues relating to illness and health. In addition, the Framework also defines the profile of the bachelor and the learning outcomes of the bachelor programme in medicine. (Laan RFJM, Leunissen RRM, Herwaarden CLA van. The 2009 framework for undergraduate medical education in the Netherlands. Netherlands Journal of Medical Education 2010;29(1):10-15)
\end{abstract}

\section{Introduction}

In February 2007, the Board and the Assembly of Deans of Medical Schools of the Dutch Federation of University Medical Centres decided to institute a Project Group to revise the 2001 Medical Education Blueprint. The main reasons for undertaking this project were developments in medical scientific disciplines, such as biotechnology and genetics, and developments in medical education, such as the introduction of the bachelor-master structure and the ongoing modernization of postgraduate speciality training programmes. In revising the 2001 Blueprint the project group was specifically asked to establish the attainment levels of both the bachelor and the master programme in medicine, to consider whether the CanMEDS $^{1}$ model or a similar competency profile could also be serviceable in revising the new Framework for medical education and to reconsider the level of detail of the revised 2001 Blueprint, aiming to find formulations that are realistic and testable and that facilitate external accountability for programme content. (It was decided to change the terminology from 'Blueprint' to 'Framework'. The term 'Framework' was thought to reflect bettter the nature of the document).

On August 12, 2009, the 2009 Framework for Undergraduate Medical Education in the Netherlands was presented to the Dutch minister for Health. A translation in English is available from the website of the Dutch Federation of University Medical Centres (http://www.nfu.nl/file admin/documents/Raamplan2009engelstal ige_versie.pdf).

\footnotetext{
* The project group consisted of professor C.L.A. van Herwaarden (chairperson), professor R.F.J.M. Laan (project coordinator), drs. R.R.M. Leunissen (secretary), dr. W.M.C. Mulder, dr. H. de Vries, professor F.G.M. Kroese, professor J.H. Bolk, professor B.G.M. van Engelen, professor A.C. Nieuwenhuijzen, professor T.J.M. Helmerhorst, professor E.A.M Sanders and professor N.S. Klazinga. Advisory members were dr. L. Wigersma (Royal Dutch Medical Association), dr. M.A.G. van den Berg (Clients Council of University Medical Centres), drs. L. Schöffer (National Medical Students Platform), dr. R.A.F. de Lind van Wijngaarden (National Interns Platform) and D.I.M. Hoefnagel LL.M (Ministry of Health, Welfare and Sports).
} 
The 2009 Framework consists of nine chapters:

1. Introduction

2. Explanation

3. Developments in Dutch and European Legislation

4. Stages, Cycles and Levels in Medical Education in the Netherlands

5. Master's Degree Programme in Medicine: Target Profile

6. Master's Degree Programme in Medicine: Competencies

7. Master's Degree Programme in Medicine: Issues Relating to Illness and Health

8. Bachelor's Degree Programme in Medicine: Profile and Learning Outcomes

9. Basic Sciences in the Medical Curriculum

In an appendix the final document also includes a skills list.

\section{The structure of medical education in the Netherlands}

In 2002, the bachelor-master structure was introduced in the Dutch system for higher education, establishing bachelor's degree and master's degree programmes as independent study programmes. This change followed in the wake of the signing of the Bologna Declaration in 1999. This Declaration, signed by 29 European countries, aims to promote greater overall convergence in higher education in Europe, based on a two-cycle degree structure. In the transitional period, two-cycle and one-cycle programmes have been available simultaneously.

Permission to use the protected title of physician and qualification to perform certain actions reserved for physicians are linked to registration in the Individual Health Care Professions Act register. Registration is only open to those who have completed the degree programme in med- icine and therefore to those who, following the introduction of the bachelor-master structure, have obtained the Master of Science (MSc) degree on completion of the master's degree programme in medicine.

\section{Important choices in the 2009 framework}

The Project Group decided to define students' learning outcomes upon completion of the master's degree programme in terms of competencies. A competency is defined as the ability to adequately perform a professional activity in a specific, authentic context, using an integrated body of knowledge, understanding, skills and professional behaviour. After careful consideration, the CanMEDS model was chosen, because, firstly, this model involves an excellent and useful division into physicians' roles and competencies in a variety of professional situations, and secondly, this model is also used in the framework of the modernization of postgraduate specialty training. Together, the seven roles or competency domains in the CanMEDS model constitute the target profile in chapter 5 of the 2009 Framework (Table 1). In chapter 6 , the profile is elaborated in terms of key competencies and their constituent sub-competencies.

Table 1. Physician' roles included in the Profile.
1. Medical Expert
2. Communicator
3. Collaborator
4. Manager
5. Health Advocate
6. Scholar
7. Professional

As the Project Group felt that bachelor students in medicine do not yet operate in 
an authentic professional setting, the bachelor's degree programme is described in terms of knowledge, skills, and behaviour and not competencies. Knowledge and understanding relate to the basic sciences, the foundation of medicine in the natural sciences, and aspects of behavioural and social sciences.

The 2001 Blueprint included a list of clinical conditions which comprised about 330 clinical conditions in all. The Project Group wanted to trim down this number to a more manageable set. Having considered the issue at length, the Project Group concluded that the phrase 'issues relating to illness and health' was more appropriate to the wide-ranging field in which today's physicians are operating, as a fair share of physicians' work is not directly patient or disease related. The list of issues described in chapter 7 has been subdivided into several categories. The first and most voluminous one comprises the complaints that induce patients to consult their physician. The next two, smaller, categories deal with findings upon physical and additional examinations. The final category comprises a list of so-called healthcare issues.

The introduction of a list of issues meant that the list of clinical conditions that was included in the 2001 Blueprint was not to return in the 2009 Framework: on the one hand, the contemplation of each issue would involve a series of clinical presentations anyway, and, on the other hand, a list of clinical conditions would require ceaseless maintenance in the wake of ongoing developments in medical disciplines.

In the 1994 Blueprint and the 2001 Blueprint, the domain of knowledge only came up to a fairly limited extent. The Project Group felt that the aspect of required knowledge had been somewhat eclipsed by lists of problems, clinical con- ditions and skills. Especially the basic sciences had remained underexposed, despite their importance. Right from the start, the Project Group wished to give the basic sciences a more prominent place in medical education. The model of competencies selected by the Project Group also carried the risk of outlining required knowledge only implicitly. Therefore, in the 2009 Framework, a separate chapter is devoted to basic sciences in the medical curriculum dealing with both the foundation of medicine in the natural sciences and aspects of the behavioural and social sciences.

The Appendices to the 2001 Blueprint included a skills list. Spot-checking at the eight Dutch medical faculties showed that all medical faculties still used this list, sometimes with minor adjustments. After discussion in the Project Group, the skills list from the previous Blueprint, with minor adjustments, was added to the Appendices to the 2009 Framework.

\section{Stages, Cycles and Levels in Medical Education in the Netherlands}

The Project Group was asked to pay special attention to finding formulations that are realistic and testable and that allow external accountability for medical education programme content. This prompted the Project Group to clearly define the competencies in terms of levels that are to be attained.

Medical education in the Netherlands involves a training sequence with two stages that medical students pass through in succession. The first stage of this training sequence comprises two cycles: a three-year bachelor's degree programme in medicine, followed by a three-year master's degree programme in medicine. In the second stage of the training sequence, following upon completion of the bache- 
lor and master programmes, medical graduates undertake postgraduate specialist training to become medical specialists in primary or secondary healthcare. Having completed that stage, medical practitioners then engage in lifelong learning activities both in practice and in formal continuing education courses.

The levels linked to the successive stages can be called starter level, beginning practitioner level and experienced practitioner level.

After completion of their bachelor programme in medicine, medical students can be characterized as starters. These students have mastered a basic body of knowledge and understanding of scientific disciplines that are relevant to their subsequent professional practice. They have also acquired a set of basic skills and are able to show appropriate professional behaviour in training-related situations. They are able to apply knowledge, skills and behaviour in dealing with issues that involve a relatively low level of complexity.

After completion of the master programme in medicine, medical graduates can be characterized as beginning practitioners. Newly graduated physicians demonstrate a basic competence in practice, possess an integrated body of knowledge, skills and professional behaviour and can handle issues involving higher levels of complexity. Medical graduates are capable of performing independent consultations but only perform these under supervision during postgraduate medical training.

After a number of subsequent years of further schooling, training and experience in medical specialist training in the discipline of their choice, physicians operate independently without supervision. They are then experienced practitioners or medical specialists. An element of inde- pendent practice is participation in interprofessional coaching activities and lifelong continuing-education courses.

In the 2009 Framework, the levels of proficiency to be achieved in the bachelor and master programmes in medicine are defined in a five-level structure (Table 2).

In discussing the levels that must be attained, the Project Group also had to consider the level of difficulty of the issues that are presented to students. This level of difficulty is chiefly determined by the presence of contextual factors. On the one hand, these are medical factors, such as typical and atypical presentations or comorbidity problems; on the other hand, these are contextual factors from the psychosocial model. The level of difficulty is then codetermined by the availability of standard solutions to a problem and the applicability of protocols and guidelines. The level of difficulty also lies in the degree to which different physician roles must be integrated in dealing with the issue in question. Graduates in medicine must be able to handle issues involving varying including higher - levels of difficulty, with the proviso that the degree of required supervision increases with the degree of difficulty of the case presented. In this respect, there is one sub-competency incorporated in the role of medical expert that is of particular importance: students' ability to recognize and name the personal limits to their knowledge and skills and to decide in time if, and, if so, when a third party needs to be called in, including their supervisor.

The 2009 Framework states that graduates in medicine must have mastered all issues related to illness and health that are included in the list at least at level II. Some of the issues, depending on the specifics of individual training programmes, will be included in additional competency testing at higher levels. The 
Table 2. Levels defined up to the beginning practitioner level.
Level I A Students have knowledge and understanding of those branches of science that are relevant to medicine.
B Students demonstrate in standardized situations that they have skills that are relevant to medicine.
C Students demonstrate that they possess the basic skills required for professional behaviour.

Level II Students use integrated knowledge, skills, and professional behaviour in an adequate* approach to the issues of illness and health incorporated in this Framework. They demonstrate such competence in context-rich training situations.

Level III Students adequately* perform the professional activities defined in the physicians' competencies in purpose-designed training situations and/or in simulated professional situations.

Level IV Students adequately* and independently perform the professional activities defined in the physicians' competencies in authentic professional situations, having received prior case-specific instruction and being intensively supervised by an experienced practitioner.

Level V Students adequately* and independently perform the professional activities defined in the physicians' competencies. An experienced practitioner is immediately available on stand-by and always provides supervision after the event.

\footnotetext{
* The performance of professional activities is considered adequate if such performance is in line with the current state of science and the prevailing standards and guidelines of the profession. Wherever the Framework mentions activities that, as a rule, span longer periods of time, as in guiding the chronically ill, students demonstrate their competency by adequately performing partial activities.
}

Table 3. Examples of (sub)competencies and levels assigned per physician's role.

\begin{tabular}{llc}
\hline Physician's role & Competency (Medical graduates are able to ...) & Level \\
\hline Medical expert & $\begin{array}{l}\text { take a medical history } \\
\text { draft a plan for diagnosis and / or therapy } \\
\text { implement a diagnostic and / or therapeutic plan }\end{array}$ & V \\
Communicator & guide the chronically or incurably ill and guide patients in palliative care & III \\
Collaborator & $\begin{array}{l}\text { show an understanding of group processes and their impact on the } \\
\text { healthcare process }\end{array}$ & V \\
Manager & apply a quality model in practice if this is relevant & III \\
Health Advocate & apply knowledge of epidemiology in practice at the individual patient level & V \\
Scholar & draft clinical protocols for the benefit of patient care & III \\
Professional & $\begin{array}{l}\text { accept responsibility for their own professional performance } \\
\text { terminate a doctor-patient relationship in a professional way }\end{array}$ & V IV \\
\hline
\end{tabular}


formulations of sub-competencies specify the levels (III, IV, or V) that have to be attained. To illustrate the assignment of levels some examples are given in Table 3.

\section{Conclusion}

The 2009 Framework differs substantially from the 2001 Blueprint. Major changes are the definition of competencies, the indication of the levels of competency to be attained and the introduction of a list of issues related to health and disease. The knowledge domains of basic sciences relevant to medicine are described in more detail. The 2009 Framework also includes the attainment levels of the bachelor programme in medicine. The 2009 Framework will be used as the basis for accreditation and for a revision of the legislation that outlines the educational requirements of physicians.

\section{References}

1. Jason R. Frank, MD MA (Ed) FRCPC. The CanMEDS 2005 Physician Competency Framework: Better standards. Better physicians. Better care. The Royal Colege of Physicians and Surgeons of Canada; 2005.

\section{The authors:}

R.F.J.M. Laan, MD PhD, is professor of rheumatology and medical education, Radboud University Medical Centre, Institute for Medical Education, Nijmegen, the Netherlands.

R.R.M. Leunissen, MSc, is senior staff member, Radboud University Medical Centre, Institute for Medical Education, Nijmegen, the Netherlands.

C.L.A. van Herwaarden, MD PhD, is professor of pulmonology and former dean of the Radboud University Medical Centre, Nijmegen, the Netherlands.

\section{Correspondence}

Prof. dr. R.F.J.M. Laan, Radboud University Medical Centre, P.O. Box 9101, 6500 HB Nijmegen, the Netherlands.E-mail:r.laan@reuma.umcn.nl

No potential conflict of interest relevant to this article was reporte 